\title{
RELAÇÃO ENTRE DOENÇA PERIODONTAL E PROTEÍNA C-REATIVA EM PACIENTES COM DOENÇA CARDIVASCULAR - REVISÃO DE LITERATURA
}

\section{RELATIONSHIP BETWEEN PERIODONTAL DISEASE AND C-REACTIVE PROTEIN AMONG PATIENTS WITH CARDIOVASCULAR DISEASES - A LITERATURE REWIEW}

\author{
Fabrício Rutz da Silva1, Fábio Aníbal Jará Goiris², Gibson Luiz Pilatti ${ }^{3}$, \\ Fábio André dos Santos ${ }^{3}$ \\ 1 Autor para contato: Cirurgião-dentista, Ponta Grossa, Paraná, Brasil; \\ e-mail: fabriciorutz@bol.com.br \\ 2 Universidade Estadual de Ponta Grossa - UEPG, Departamento de Odontologia, \\ Campus em Uvaranas, Ponta Grossa, PR \\ 3 Universidade Estadual de Ponta Grossa - UEPG, Programa de Pós-Graduação em \\ Odontologia - Mestrado, Campus em Uvaranas, Ponta Grossa, PR
}

Recebido para publicação em 10/12/2007

Aceito para publicação em 13/02/2008

\section{RESUMO}

Vários estudos demonstram uma forte associação entre a doença periodontal e as doenças cardiovasculares. Embora ainda não exista um consenso entre os pesquisadores a respeito desta ligação, as alterações na resposta imune causadas pela carga microbiológica contínua parecem ser a chave para a elucidação do problema. Partindo dessa afirmação, vários estudos têm procurado mensurar os níveis plasmáticos de alguns mediadores da inflamação, em pacientes com doença periodontal, antes e após tratamento, relacionando os resultados com o risco de desenvolvimento de doenças cardiovasculares. Um dos marcadores inflamatórios já classicamente relacionado com doenças cardiovasculares éa proteína C-reativa (PCR).

Palavras-chave: doenças periodontais, doenças cardiovasculares, reação de fase aguda, proteína C-reativa. 


\begin{abstract}
Several studies have demonstrated a strong association between periodontal and cardiovascular diseases. Although there is no consensus among researchers in relation to this association, alterations in the immune response caused by the continuous action of periodontal bacteria seem to be the key factor to elucidate this dilemma. Therefore, several studies have been trying to measure the plasmatic levels of some inflammatory mediators in patients with a periodontal disease. CReactive Protein (CRP) is an inflammatory marker related with cardiovascular diseases. Its concentration could indicate acute inflammation. In the present work, the relationship between periodontal diseases and cardiovascular alterations and their mechanisms is discussed. The variations of CRP and their role in periodontal disease and how inflammatory markers contribute to identify patients with a high a risk of developing cardiovascular diseases are also analized, and thus become a form of predicting risk of cardiovascular problems.
\end{abstract}

Key words: periodontal diseases, cardiovascular diseases, acute-phase reaction, C-reactive protein.

\section{Introdução}

Apesar de novas especialidades médicas e odontológicas surgirem, não existe como tratar um paciente apenas conhecendo uma determinada área, isso pode ser observado tanto na Medicina, quanto na Odontologia (Silva, 2004). Um tratamento holístico, ou seja, que veja o indivíduo como um todo, é ensinado com ênfase nos cursos de graduação. Baseado nisso, é natural que especialidades da Medicina e Odontologia se inter-relacionem. Com certeza a Cirurgia e Traumatologia Buco-maxilo-facial é o maior exemplo disso. Porém, tem surgido uma nova área chamada de Periodontia Médica ou Medicina Periodontal que, a cada dia, através de inúmeros trabalhos científicos, tem se firmado como um ramo importante das ciências da saúde. (FERREIRA, 1997; SILVA, 2004).

Segundo Williams e Offenbacher(2000), o termo Medicina Periodontal é um novo ramo da Periodontia que estabelece a relação entre as doenças sistêmicas e as doenças periodontais. Esta relação teria dois caminhos: saúde ou doença periodontal influenciando a saúde sistêmica ou em um sentido inverso, a saúde periodontal sendo influenciada por condições sistêmicas.

Entre as várias enfermidades sistêmicas que poderiam ter seu risco aumentado devido a problemas periodontais, as doenças do coração têm papel de destaque (BECK et al., 1996; KINANE e LOWE, 2000; LOSS et al., 2000; EMINGIL et al., 2000; SAITO et al., 2003; OKUDA et al., 2004; D’AIUTO et al., 2004).

Um diagnóstico precoce é essencial para um tratamento efetivo. Para fazer esse diagnóstico muitas vezes são usados os marcadores de risco. Marcadores de risco podem ser definidos como características biológicas cuja presença ou nível indica um maior risco de desenvolvimento de uma doença em particular. Em determinado instante, esses fatores podem ter um papel causal no desenvolvimento de doenças, bem como modificações para redução do risco. Outros fatores podem ser acidentais para o processo etiológico, servindo como bons indicadores do risco de doença, ou resposta ao tratamento (MAGLIANO et al., 2003).

Segundo Magliano et al. (2003), a proteína Creativa (PCR) é o mais promissor dos novos marcadores de risco para doenças cardiovasculares. Ela tem uma forte associação com risco de doença cardiovascular, com segurança e baixo custo. PCR é uma proteína de fase aguda, sendo produzida no fígado em resposta a estímulos inflamatórios. Em vários estudos (RIDKER et al., 1998; JAGER et al., 1999), PCR de 
alta sensibilidade tem sido o fator de risco independente mais forte para predizer infarto, comparado com tradicionais fatores de risco, e um melhor preditor de risco que níveis equivalentes de hemocisteína.

A doença periodontal é uma patologia que gera uma resposta inflamatória crônica de baixa intensidade, aumentando os níveis de PCR. Assim, são vários os trabalhos que investigam o papel dessas alterações e sua relação com os problemas cardiovasculares (Loss et al., 2000; Davis, 2002; SAITO et al., 2003; D’AIUTO et al., 2004).

\section{Revisão de Literatura}

Dividiremos a revisão de literatura em duas partes: trabalhos associando doença periodontal e doença cardiovascular e estudos que tratam das variações nos níveis da proteína C-reativa (PCR) em pacientes com doença periodontal.

\section{Doença periodontal e doença cardiovascular}

O primeiro trabalho associando doença periodontal e doença cardiovascular foi publicado em 1963, por Mackenzie e Millard. Este estudo investigou a relação entre diabetes e aterosclerose com perda óssea alveolar. Foram avaliados 60 indivíduos diabéticos, 64 com suspeita de diabetes, 54 com aterosclerose e sem diabetes e um grupo controle de 74 pacientes sem diabetes e aterosclerose. O resultado mostrou que 62\% do grupo com aterosclerose tiveram mais perda óssea do que o grupo controle.

Emingil et al. (2000), investigaram a possível associação entre saúde periodontal e doença coronariana em pacientes com infarto agudo do miocárdio e doença crônica do coração. Um total de 120 pacientes, sendo 60 com infarto do miocárdio e 60 com doença coronariana crônica participaram do estudo. No exame clínico foi observado o número de dentes perdidos, restaurações, profundidade de sondagem e sangramento à sondagem. Amostras de sangue foram coletadas na admissão para medidas do colesterol total, triglicerídeos, HDL, LDL e nível de glicose em jejum. A percentagem de sítios com sangramento à sondagem, número de sítios com profundidade clínica de sondagem e” 4mm, número de restaurações, freqüência do fumo e os níveis de triglicerídeos no sangue foram significativamente associados com infarto agudo do miocárdio. Assim, os autores concluíram que a doença periodontal pode ser associada ao infarto agudo do miocárdio.

Em 2001, Jansson et al. estudaram a relação entre saúde periodontal e doença cardiovascular fatal. Utilizaram dados referentes a 1393 pessoas, de 18 a 66 anos, examinadas em um estudo epidemiológico entre 1970 e 1971. O período de acompanhamento foi de 1970 até 1996 . Os resultados mostraram que 27\% dos homens e 20\% das mulheres morreram. Em 49\% das mortes, a doença cardiovascular causou ou contribuiu para a causa da morte. A prevalência de doença cardiovascular na amostra em 1970 foi de 7\% e 18\% dos indivíduos morreram entre 1970 e 1996 desta doença. A conclusão é de que doenças bucais foram colocadas como um fator de risco para morte por doença cardiovascular, especialmente em combinação com outros fatores de risco conhecidos como fumo.

Em 2002, Buhlin et al. avaliaram a relação entre saúde dental e doença cardiovascular na população adulta da Suécia. Um questionário sobre hábitos de cuidados dentais, doenças cardiovasculares e situação sócio-econômica foi enviado para 4811 suecos. Probabilidade média para todas as doenças cardiovasculares e o subgrupo infarto do miocárdio, derrame e hipertensão foi calculada pelo modelo de regressão logística ajustado para idade, gênero, fumo, renda, estado civil e educação. Essas médias foram calculadas apenas para indivíduos com idades $\geq$ a 41 anos, porque, segundo os autores, poucas pessoas têm doença cardiovascular antes disso. Foi encontrada significativa associação entre o auto-relato de sangramento gengival, presença de prótese total e doenças cardiovasculares. Entretanto, não houve associação entre perda dentária, profundidade de bolsas e doenças cardiovasculares. Este estudo demonstra associação entre doença cardiovascular e sangramento gengival e presença de próteses removíveis. Entretanto não indica a natureza dessa associação.

Madianos et al. (2002), fizeram uma revisão sistemática de vários trabalhos objetivando avaliar a associação entre a doença periodontal e o aumento do risco de doença cardiovascular. Os autores analisaram 8 estudos coorte, 4 casos controle e 4 transversais. 
Trabalhos de meta-análise não foram incluídos devido a grande heterogeneidade dos estudos, principalmente com relação ao mensuramento das condições periodontais. Em 50\% dos estudos coorte (4/8), 75\% dos estudos caso-controle (3/4) e 50\% dos estudos transversais (2/4) mostraram uma significativa associação entre medidas clínicas de periodontite e doenças cardiovasculares. A conclusão foi de existe uma associação entre doença periodontal e doença cardiovascular, sendo necessário novos estudos para confirmação dessas observações, estabelecendo a causa e determinando os potenciais benefícios do tratamento periodontal na redução do risco dessas condições.

\section{Proteína C-Reativa (PCR)}

Torna-se de especial interesse para a Periodontia, as proteínas de fase aguda induzidas por IL-1, principalmente a proteína C-reativa. A mesma funciona opsonizando células cobertas por polissacarídeos.

De acordo com Oppermann e Rösing (2001), apesar da necessidade de futuras confirmações, a PCR parece ser um interessante marcador plasmático para a fase aguda da periodontite. O exame imunológico é feito no plasma do paciente e os resultados podem ser dados qualitativamente ou quantitativamente. O método qualitativo diz se o paciente é reativo ou não, sendo o resultado não reativo utilizado como valor de referência. O método quantitativo é mais sensível. O valor de referência é de até $0,5 \mathrm{mg} / \mathrm{dL}$.

Fredriksson et al., em 1999, estudaram o efeito da periodontite e fumo nos leucócitos do sangue e proteínas da fase aguda. Amostras de sangue venoso foram retiradas de 40 adultos com periodontite (20 mulheres, 20 homens; média de idade $52.4 \pm 8.4$ ) e 43 indivíduos saudáveis controles ( 25 mulheres, 18 homens; média de idade $50.6 \pm 7.6)$. Os pacientes do grupo teste tinham 6 sítios com perda de inserção clínica de $\geq 5 \mathrm{~mm}$, sendo que o grupo controle, tinha perda de inserção clínica $<3 \mathrm{~mm}$. Os resultados demonstraram um aumento na atividade dos neutrófilos periféricos em pacientes com periodontite. $O$ fumo não teve um efeito significativo nesse aumento de atividade. Os pacientes do grupo teste tinham mais altas concentrações de PCR do que o controle. Os autores concluíram que a periodontite por si só induz alterações nos leucócitos e nas proteínas de fase aguda, entre elas a PCR. Quando a periodontite e o fumo são associados, essas alterações são mais pronunciadas.

Em 2001, Di Napoli et al., avaliaram os níveis de PCR após acidentes vasculares cerebrais. A reação inflamatória causada por um acidente vascular cerebral pode ser medida pela determinação da concentração da PCR. Este estudo avaliou os níveis dessa proteína em 193 pacientes, sendo 72 homens e 121 mulheres (73 \pm 9 anos) que foram hospitalizados por acidente vascular cerebral. Os níveis da PCR foram mensurados nos seguintes momentos: no internamento, 24 horas e 48 horas após o acidente vascular cerebral e na alta hospitalar. Os níveis da PCR encontrados foram: 12 mg/L nos pacientes sobreviventes na admissão hospitalar, $28 \mathrm{mg} / \mathrm{L}$ nos que morreram após admissão hospitalar, 13 mg/L após 24 horas, $10 \mathrm{mg} / \mathrm{L}$ após 48 a 72 horas e 6 mg/L na alta hospitalar. A conclusão dos autores foi de que os pacientes com acidentes vasculares e que tinham reações inflamatórias mais intensas tiveram maior risco de morte. A doença periodontal e a PCR podem ser consideradas como marcadores, porque podem identificar o grupo de pacientes de alto risco.

Em 2003, Iwamoto et al., examinaram os efeitos do tratamento periodontal nos índices de PCR, adiponectina e fator de necrose tumoral á (TNF $\alpha$ ). Participaram do estudo 15 pacientes (10 homens e 5 mulheres), com periodontite crônica com várias condições sistêmicas de alto risco para aterosclerose (diabetes tipo 2, hipertensão arterial). Os pacientes foram tratados com terapia não cirúrgica com aplicações tópicas de antibióticos (10 mg de minociclina-HCl/bolsa) e remoção do cálculo, com ultra-som. Antes e depois do tratamento, PCR, adiponectina e TNF $\alpha$ foram medidos. Os resultados demonstraram que os níveis de PCR e TNF á foram significativamente menores após o tratamento, ao passo que níveis de adiponectina não sofreram mudanças significativas. Assim, concluise que o tratamento periodontal foi efetivo na redução de PCR e TNF á, mas não de adiponectina.

\section{Discussão}

Embora ainda não esteja claro o real mecanismo da associação entre doença periodontal e problemas 
cardiovasculares, a grande maioria dos trabalhos demonstra uma forte ligação entre esses dois grupos de patologias. A resposta inflamatória crônica e de baixa intensidade causada pela doença periodontal parece ser a chave para o entendimento dessa associação (BECK et al., 1996; KINANE e LOWE, 2000; LOSS et al., 2000; SAITO et al., 2003; OKUDA et al., 2004; D'AIUTO et al., 2004).

Beck et al. (1996) relacionando a doença periodontal com as doenças cardiovasculares, hipotetizaram que sendo a doença periodontal, uma infecção crônica por microorganismos Gram-negativos, representaria um prévio fator de risco para aterosclerose e eventos tromboembolíticos. Após estabilizada, a doença periodontal promoveria uma sobrecarga biológica de endotoxinas (LPS) e citocinas inflamatórias (especialmente Tx2, IL $1 \beta$, PGE2 e TNF- $\alpha$ ) que iniciariam uma exacerbada aterogênese e eventos tromboemolíticos.

Kinane e Lowe (2000) ressaltam em seu trabalho, o papel imunológico dos microorganismos periodontopatogênicos como coadjuvantes no início da aterosclerose. O início da aterosclerose se dá através do acúmulo local de lipídios e outras proteínas plasmáticas na camada intima das artérias. Os monócitos aderem e penetram no endotélio durante os estágios iniciais da formação da placa ateromatosa, ajudados pela adesão molecular, por citocinas quimiotáticas e citocinas pró-inflamatórias. Essas células tornam-se enfartadas com o colesterol de baixa densidade (LDL). Os monócitos transformam-se em macrófagos dentro das lesões, causando destruição, pela liberação de enzimas, guiando a trombose ou aumentando a placa pela liberação de fatores mitóticos como fator de crescimento derivado de plaquetas. Produtos da degradação da fibrina parecem estar envolvidos nos eventos de trombose, quimiotaxia e propriedades mitóticas que aumentam a propagação da lesão e a proliferação de células musculares, com a produção de uma capa fibromuscular debaixo do endotélio e subseqüente isquemia. A aderência de monócitos no endotélio, após sua migração debaixo da camada intima do endotélio, é considerado como fator inicial da formação do ateroma.

Além de causarem alterações na resposta imune, os microorganismos podem atuar de foram direta nas doenças cardiovasculares. Bactérias, como Porphyromonas gingivalis, Actinobacillus actinomycetemcomitans, Campylobacter rectus, Bacteroides forsythus e Treponema denticola, foram encontradas em placas ateromatosas (KINANE e LOWE, 2000).

Sabe-se que o paciente com doença periodontal está submetido constantemente à bacteremia, mesmo durante atos simples como a escovação ou a mastigação. Assim o paciente periodontal crônico tem constantemente em sua corrente sangüínea microorganismos que podem colonizar vários pontos de seu organismo, principalmente onde existam variações anatômicas (KUNZE, 1999).

Vários trabalhos demonstram que pacientes com doença periodontal possuem níveis elevados de PCR no sangue (FREDRIKSSON et al., 1999; LOSS et al., 2000; NOACK et al., 2001; DAVIS, 2002; IWAMOTO et al., 2003; CRAIG et al., 2003; SAITO et al., 2003; D’AIUTO et al., 2004). Assim, a avaliação dos níveis de PCR pode ser mais uma arma no diagnóstico e no acompanhamento da evolução da doença periodontal, mas não apenas isso. Segundo Saito et al. (2003), a inflamação tem um papel importante na aterosclerose, o que poderia levar ao desenvolvimento de outras doenças cardiovasculares. Com a evolução dos estudos sobre a relação entre a doença periodontal e doenças sistêmicas, a mensuração dos níveis de PCR assume um papel importante também na avaliação do risco do paciente vir a desenvolver problemas cardiovasculares (LOSS et al., 2000; DAVIS, 2002; SAITO et al., 2003; D’AIUTO et al., 2004).

Alguns estudos sugerem a avaliação de outro marcador sistêmico da inflamação: a IL-6 (LOSS et al, 2000; D’AIUTO et al., 2004).

\section{Conclusão}

Com base na revisão de literatura podemos concluir que a grande maioria dos trabalhos indica uma relação positiva entre doença periodontal e doença cardiovascular.

\section{REFERÊNCIAS}

1. BECK, J.; GARCIA, R.; HEISS, G.; VOKONAS, P. S.; OFFENBACHER, S. Periodontal disease and cardiovascular 
disease. J Periodontol, Chicago, v. 67 Suppl, p. 1123-1137, 1996.

2. BUHLIN, K.; GUSTAFSSON,A.; HÅKANSSON, J.; KLINGE, B. Oral health and cardiovascular disease in Sweden. J Clin Periodontol, Copenhagen, v.29, p. 254-259, 2002.

3. CRAIG, R. G.; YIP, J. K.; SO, M. K.; BOYLAN, R. J.; SOCRANSKY, S. S.; HAFFAJEE, A. D. Relationship of destructive periodontal disease to the acute-phase response. J Periodontol, Chicago, v. 74, 1007-1016, 2003.

4. D’AIUTO, F.; READY, D.; TONETTI, M. S. Periodontal disease and C-reactive protein-associated cardiovascular risk. J Periodontol. Res; Copenhagen, v. 39, p. 236-241, 2004.

5. DAVIS, R. B. C-reactive protein and bacterial DNA in the blood of cardiac patients with periodontitis [abstract]. J Periodontol, Chicago, v. 73, n. 8, p. 970-971, 2002.

6. DI NAPOLI, M.; PAPA, F.; BOCOLA, V. Periodontal disease, C-reactive protein, and ischemic stroke. Arch Intern. Med, Chicago, v. 161, n.9, 1234-1235, 2001.

7. EMINGIL, G.; BUDUNELI, E.; ALIYEV, A.; AKILLI, A.; ATILLA, G. Association between periodontal disease and acute myocardial infarction. J Periodontol, Chicago, v. 71, p. 18821886, 2000.

8. FERREIRA, R. A. De quem é o bisturi? Rev Assoc Paul Cir Dent, São Paulo, v. 51, n. 1, 9-19, 1997.

9. FREDRIKSSON, M. I.; FIGUEREDO, C. M. S.; GUSTAFSSON, A.; BERGSTRÖM, K.G.; ÅSMAN B. Effect of periodontitis and smoking on blood leukocytes and acutephase proteins. J Periodontol, Chicago, v. 70, p. 1355-1360, 1999.

10. IWAMOTO, Y. et al. Antimicrobial periodontal treatment decreases serum C-reactive protein, tumor necrosis factor-alfa, but not adiponectin levels en patients with chronic periodontitis. J Periodontol, Chicago, v. 74, p. 1231-1236, 2003.

11. JAGER A.; VAN HINSBERH, V. W.; KOSTENSE, P. J.; EMEIS, J. J.; YUDKIN, J. S.; NIJPELS, G et al. Von Willebrand factor, C-reactive protein, and 5-year mortality in diabetic and nondiabetic subjects: the Hoorn Study. Arterioscler Thromb Vasc Biol, Dallas, v. 19, p. 3071-3078, 1999.

12. JANSSON, L.; LAVSTEDT, S.; FRITHIOF, L.; THEOBALD, H. Relationship between oral health and mortality in cardiovascular diseases. J Clin Periodontol, Copenhagen, v. 28, p. 762-768, 2001.

13. KINANE, D. F., LOWE, G. D. O. How periodontal disease may contribute to cardiovascular disease. Periodontology 2000, Copenhagen, v. 23, p. 121-126, 2000.

14. KUNZE, B. J. C. A doença periodontal como fator de risco para aterosclerose e doença cardíaca coronariana. [Monografia] EAPABO-PR/PG 1999.
15. LOOS, B. G.; CRAANDIJk, J.; HOEK, F. J.; PAULIEN, M. E.; DILLEN, W.; VELDEN, U. Elevation of systemic markers related to cardiovascular diseases in the peripheral blood of periodontitis patients. J Periodontol, Chicago, v. 71, n. 10, p.1528-1534, 2000.

16. MACKENZIE, R. S.; MILLARD, H. D. Interrelated effects of diabetes, arteriosclerosis and calculus on alveolar bone loss. J Am Dent Assoc, Chicago, v. 66, p. 191-199, 1963.

17. MADIANOS, P. N.; BOBETSI, G. A.; KINANE, D. F. Is periodontitis associates with an increased risk of coronary heart disease and preterm and/or low birth weight births? J Clin Periodontol, Copenhagen, v. 29, Supp 13, p. 22-36, 2002.

18. MAGLIANO, D.J.; LIEW, D.; ASHTON, E. L.; SUNDARARAJAN, V.; MCNEIL, J. Novel biomedical risk markers for cardiovascular disease. J Cardiovasc Risk, London, v.10, p. 41-54, 2003.

19. NOACH, B.; GENCO, R. J.; TREVISAN, M.; GROSSI, S.; ZAMBON, J. J.; NARDIN, E. Periodontal infections contribute to elevated systemic C-reactive protein level. J Periodontol, Chicago, v. 72, p. 1221-1227, 2001.

20. OKUDA, K.; KATO, T.; ISHIHARA, K. Involvement of periodontopathic biofilm in vascular diseases. Oral Diseases, Copenhagen, v. 10, p. 5-12, 2004.

21.OPPERMANN, R. V. e RÖSING, C. K. Periodontia: ciência e clínica. 1. ed. São Paulo: Artes Médicas 2001, 458 p.

22. RIDKER, P. M.; CUSHMAN, M.; STAMPFER, M. J.; TRACY, R. P.; HENNEKENS, C. H. Plasma concentration of Creactive protein and risk of developing peripheral vascular disease. Circulation, Dallas, v. 97, p. 425-428, 1998.

23. RIDKER, P. M.; GLUNN, R. J.; HENNEKENS, C. H. Creactive protein adds to the predictive value of total and HDL cholesterol in determining risk of first myocardial infarction. Circulation, Dallas, v. 97, p. 2007-2011, 1998.

24. SAITO, T.; MURAKAMI, M.; SHIMAZAKI, Y.; OOBAYASHI, K.; MATSUMOTO, S.; KOGA, T. Association between alveolar bone loss and elevated serum C-reactive protein in japanese men. J Periodontol, Chicago, v. 74, p.17411746, 2003.

25. SILVA, S. R. Medicina periodontal: a arte da integração Rev Assoc Paul Cir Dent, São Paulo, v. 58, n. 1, p. 7-17, 2004.

26. WILLIAMS, R. C.; OFFENBACHER, S. Periodontal medicine: the emergence of new branch of periodontology. Periodontology 2000, Copenhagen, v. 23, p. 9-12, 2000. 